\title{
A modified and tailored human follicle isolation procedure improves follicle recovery and survival
}

\author{
Maria Costanza Chiti', Marie-Madeleine Dolmans ${ }^{1,2^{*}}$, Maria Hobeika ${ }^{1}$, Alice Cernogoraz ${ }^{1}$, Jacques Donnez ${ }^{3}$ \\ and Christiani Andrade Amorim ${ }^{1}$
}

\begin{abstract}
Background: Ovarian tissue cryopreservation followed by transplantation after cancer remission is the most commonly applied fertility restoration approach in very young girls and women who require immediate cancer therapy. However, clinicians strongly advise against reimplantation of one's own ovarian tissue when there is a high risk of recurrence after grafting. For these patients, development of an alternative strategy, namely a transplantable artificial ovary, offers future hope of conceiving. The first essential requirement for an artificial ovary is the set-up of a safe and effective follicle isolation procedure. Despite encouraging results with different variants of this technique, none of them take into the account the physiology and great variability in follicular density inside individual tissue fragments and between different patients. The goal of this study was to improve our previously applied follicle isolation procedure in order to develop a tailored isolation procedure for human follicles according to individual tissue properties. To this end, enzymatic digestion was divided into three time intervals in order to initially recover the first follicles to be isolated, and then further dissociate undigested fragments of tissue containing entrapped follicles.
\end{abstract}

Results: After thawing frozen human ovarian tissue using a modified and tailored follicle isolation method, already $35 \%$ of follicles were fully isolated and recovered after 30 min of enzymatic digestion. Indeed, this protocol resulted in a higher follicle yield $(p<0.01)$ and greater numbers of primordial and primary follicles $(p<0.05)$ than the previous approach. However, no significant difference was found in caspase-3-positive and Ki67-positive staining between the two isolation protocols. In addition, greater follicle quality was demonstrated. When human follicles isolated using the modified protocol were encapsulated in a fibrin matrix with high concentrations of fibrinogen and thrombin and xenografted to a SCID mouse, more follicles were found to be healthy after one week of transplantation than in a previous our study.

Conclusions: With the modified follicle isolation method, we were able to maximize the number and quality of isolated primordial and primary follicles, and develop a tailored follicle isolation procedure according to individual tissue properties. Moreover, improved follicle survival inside an artificial ovary prototype was detected after one week of xenografting.

Keywords: Ovarian follicle isolation, Xenografting, Fibrin matrix, Artificial ovary

\footnotetext{
* Correspondence: marie-madeleine.dolmans@uclouvain.be

'Pôle de Recherche en Gynécologie, Institut de Recherche Expérimentale et

Clinique, Université Catholique de Louvain, Avenue Mounier 52, bte. B1.52.02,

1200 Brussels, Belgium

${ }^{2}$ Gynecology Department, Cliniques Universitaires Saint-Luc, 1200 Brussels,

Belgium

Full list of author information is available at the end of the article
} 


\section{Background}

In recent years, we have been focusing our efforts on the development of a transplantable artificial ovary, with a view to restoring fertility in cancer patients at high risk of ovarian involvement who cannot undergo any of the currently implemented fertility preservation strategies [1-3]. Conceptually, the artificial ovary would require removal and cryopreservation of ovarian tissue before initiation of cancer treatment, as with ovarian tissue transplantation [4-6], but unlike the latter, fragments of cryopreserved ovarian tissue would be thawed and mechanically and enzymatically digested in order to isolate preantral follicles once the patient is cured. Thereafter, these follicles would be encapsulated in a threedimensional (3D) scaffold and transplanted back to the patient in order to restore her fertility [7].

It is therefore clear that the first crucial step in such a procedure is effective isolation of human preantral follicles, which should constitute a perfect balance in terms of quantity and quality. An optimal isolation procedure should allow recovery of a maximum number of follicles from the ovarian tissue, without causing them any damage. Previous reports have already demonstrated that structurally intact human follicles can be isolated [8-12] and shown to be free of malignant cell contamination, an essential requirement for future clinical application of an artificial ovary $[13,14]$. However, such protocols do not take into account the great heterogeneity of follicular density $[15,16]$ and extracellular matrix composition $[17,18]$ between patients, which inevitably influences isolation procedure success rates. Furthermore, enzymatic digestion is usually performed over a single duration of time, without considering the possible negative impact of prolonged digestion on the first fully isolated follicles. With this in mind, the aim of the present study was to improve the follicle isolation procedure in order to maximize the number and quality of isolated follicles, and develop a tailored follicle isolation approach according to each individual ovarian tissue sample.

\section{Methods}

\section{Experimental design}

Preantral follicles were isolated from human frozenthawed biopsies $(n=23)$ according to two different isolation protocols (previously established versus modified) in order to compare follicle yield and follicle viability. Thereafter, between 14 and 50 isolated follicles (from each protocol) were embedded in fibrin clots composed of $30-75 \mathrm{mg}$ of fibrinogen $(\mathrm{F})$ and $50-75 \mathrm{IU} / \mathrm{mL}$ of thrombin $(\mathrm{T})$ to compare follicle developmental stage and survival. In addition, to confirm the impact of the modified isolation protocol on the survival of healthy follicles, one fibrin clot containing 100 follicles and 100,000 ovarian stromal cells (SCs) was xenografted to the ovarian bursa of a severe combined immunodeficient (SCID) mouse for 7 days.

Thawing of frozen ovarian cortex and sample distribution Cryovials of frozen ovarian cortex from the different patients $(n=23)$ were collected from the ovarian tissue bank and thawed as previously described [19]. Thawed ovarian fragments were measured using graph paper and then equally distributed based on their size between the previously established protocol and the newly modified version.

\section{Follicle isolation}

\section{Previously applied protocol}

This protocol has already been extensively described [10] and is detailed in the Additional file 1. Briefly, it involves a single duration (75 min) of mechanical and enzymatic tissue digestion with 0.28 Wünsch units $/ \mathrm{mL}$ Liberase DH (Roche Diagnostics, GmbH, Mannheim, Germany) before follicle pick-up.

\section{Modified protocol}

In the modified version, ovarian tissue pieces were mechanically minced into fragments with a tissue chopper (McIlwain Tissue Chopper, Mickle Laboratory, Guildford, UK) and incubated in $10 \mathrm{~mL}$ of Dulbecco's phosphate-buffered saline (PBS) with $\mathrm{Ca}^{2+}$ and $\mathrm{Mg}^{2+}$ (Gibco, Thermo Fisher Scientific, Ghent, Belgium) in the presence of 0.28 Wünsch units/mL Liberase DH (Roche Diagnostics) and 8 Kunitz units/mL DNase I (Roche Diagnostics, Brussels, Belgium) in a water bath $\left(37^{\circ} \mathrm{C}\right)$ for $30 \mathrm{~min}$ with gentle agitation and pipetted every $15 \mathrm{~min}$. After $30 \mathrm{~min}$, the suspension was first filtered with a cell strainer of $70 \mu \mathrm{m}$ (Falcon, New York, USA), then $30 \mu \mathrm{m}$ (pluriSelect Life Science, Leipzig, Germany), before being inactivated with the same volume of PBS $+10 \%$ heat inactivated fetal bovine serum (HIFBS). The enzymatic digestion step was repeated with ovarian tissue fragments collected from the $70 \mu \mathrm{m}$ cell strainer, while isolated follicles from the $30 \mu \mathrm{m}$ filter were recovered by washing the nylon mesh in $3 \mathrm{~mL}$ of PBS $+10 \%$ HIFBS. Fully isolated follicles were retrieved with the help of a stereomicroscope and $130 \mu \mathrm{m}$ micropipette by two operators. Every $30 \mathrm{~min}$, the filtration and follicle recovery steps were repeated. Once $90 \mathrm{~min}$ of enzymatic digestion was reached, with virtually all ovarian tissue fragments totally dissociated, the isolation procedure was halted. It is important to mention that each filter was checked under the stereomicroscope for entrapped follicles possibly remaining in the nylon mesh, before being discarded.

\section{Ovarian SC isolation}

After follicle isolation, the remaining ovarian stromal cell suspension was filtered through sterilized $11-\mu \mathrm{m}$ nylon 
net filters (Millipore, Overijse, Belgium). A volume of $10 \mu \mathrm{L}$ of filtered solution was analyzed using Trypan blue (Sigma-Aldrich), and a Bürker chamber (VWR, Leuven, Belgium) was utilized to count cell numbers. Thereafter, the filtered solution was centrifuged at $260 \mathrm{~g}$ for $5 \mathrm{~min}$ and the pellet resuspended in PBS supplemented with $10 \%$ FBS to obtain a concentration of 100,000 cells $/ 1 \mu \mathrm{L}$.

\section{Follicle yield}

Because of the diameter of the polycarbonate needle $(130 \mu \mathrm{m})$ used to pick up follicles in both the established and optimized protocols, follicle diameter ranged between 30 and $129 \mu \mathrm{m}$. The chance of finding follicles larger than $120 \mu \mathrm{m}$ is actually very low when we perform human ovarian follicle isolation from frozen-thawed fragments of ovarian tissue. Isolated preantral follicles were retrieved and counted in both protocols in order to compare their number and quality.

\section{Follicle viability}

To assess follicle viability, 5 to 10 follicles were incubated in $50 \mu \mathrm{L}$ of PBS containing $2 \mu \mathrm{mol} / \mathrm{L}$ calcein AM and $5 \mu \mathrm{mol} / \mathrm{L}$ ethidium homodimer-I (LIVE/DEAD ${ }^{\circ}$ Viability/Cytotoxicity Kit, Molecular Probes, Leyden, the Netherlands) for $30 \mathrm{~min}$ at $37{ }^{\circ} \mathrm{C}$ in the dark [9]. The follicles were then classified into four categories (V1 to V4) depending on the percentage of dead granulosa cells (GCs) [20]: V1) follicles with all GCs viable; V2) follicles with less than $10 \%$ dead GCs; V3) follicles with $10-50 \%$ dead GCs; and V4) follicles with more than $50 \%$ dead GCs and/or a dead oocyte. V1 and V2 were considered to be live follicles, V3 and V4 were classified as dead.

\section{Fibrin clot reconstitution and follicle encapsulation}

Reconstitution and dilution of the two components ( $F$ and $\mathrm{T}$ ) of the fibrin sealant kit (Tissucol, Baxter, Lessines, Belgium) and fibrin clot formation were previously described by Paulini et al. [21]. F (100 mg/mL) was diluted in saline solution $(0.9 \% \mathrm{NaCl})$ to obtain a final concentration ranging from 30 to $75 \mathrm{mg} / \mathrm{mL}$, and $\mathrm{T}$ (500 IU/mL) was diluted in $40 \mathrm{mmol} / \mathrm{L} \mathrm{CaCl}{ }_{2}$ to achieve a concentration between 50 and $75 \mathrm{IU} / \mathrm{mL}$.

\section{Immunohistochemistry}

To evaluate follicle health status after isolation and fibrin encapsulation, we performed caspase- 3 and Ki67 immunostaining [22, 23]. The protocol is described in detail in the Additional file 1. Follicles with more than $50 \%$ of GCs and/or the oocyte positive for caspase- 3 were classified as atretic, while follicles with at least one Ki67-marked GC were considered to be growing [21].

\section{Xenografting of a fibrin clot to a SCID mouse}

In order to confirm the effectiveness of the modified protocol, a fibrin clot containing 100 follicles and 100,000 SCs was xenografted to the left ovarian bursa of a SCID mouse. Guidelines for animal welfare were approved by the Committee on Animal Research of the Université Catholique de Louvain. The mouse was anesthetized according to a previous protocol [24, 25]. A medio lateral dorsal cutaneous incision was made and the peritoneum was opened through a small hole. The ovary was exposed and the periovarian fat was gently gripped with forceps in order to stabilize the ovary and give access to the ovarian bursa. Under an operating microscope (Leica MZ6, Wetzlar, Germany), a small opening was made in the ovarian bursal membrane to introduce the fibrin clot, before closing the membrane with non-absorbable 8/0 Prolene suture (Ethicon, Johnson \& Johnson Medical, Livingston, UK). The adnexa were replaced inside the peritoneal cavity and the peritoneum and skin were closed with non-absorbable 6/0 Prolene. After surgery, anesthesia was reversed as previously described $[24,25]$. The animal was euthanized after 7 days by cervical dislocation and the graft was recovered and fixed in $4 \%$ formaldehyde.

\section{Histological analysis}

After $24 \mathrm{~h}$ of fixation in formaldehyde, the fibrin clots were embedded in paraffin for histological analysis. Each fibrin clot was cut into $5 \mu \mathrm{m}$ serial sections and every second section was stained with hematoxylin and eosin to be counted and classified according to the developmental stage of the encapsulated follicles [24].

Follicles were classified as primordial when a single layer of flattened GCs was observed surrounding the oocyte, intermediate when one layer of flattened and cuboidal GCs was detected, primary when a full layer of cuboidal GCs was identified around the oocyte, and secondary when two or more layers of cuboidal GCs were found [26].

\section{Statistical analysis}

The Wilcoxon test was used to compare follicle yield and viable follicles before encapsulation between the previous and the modified isolation protocol. Fisher's exact test was applied to compare follicle developmental stages and Ki67-positive follicles after encapsulation. A $p$-value $<0.05$ was considered statistically significant. All statistical analyses were performed with GraphPad Prism 7 software (GraphPad Software, La Jolla, USA).

\section{Results}

After isolation

Follicle yield

A total of 4661 follicles were isolated with the two protocols, 1938 with the previously applied protocol after 
75 min of digestion (Table 1, Fig. 1a), and 2723 with the modified protocol using the same sample size from the same patients (Table 1, Fig. 1b).

Moreover, in the latter case, $35 \%$ of that number were already fully isolated and recovered after $30 \mathrm{~min}$ of digestion. The remaining $65 \%$ were recovered after $60 \mathrm{~min}$ (42\%) and $90 \mathrm{~min}$ (23\%) of digestion (Fig. 1c). As already well known from the literature [16], there is great variation in follicle density between patients (Table 1). When the mean $\pm \mathrm{SD}$ of isolated follicles was compared between the two protocols, the modified version showed a significantly higher number of isolated follicles $(123.8 \pm 121.7)$ than the previous protocol $(88.1 \pm 85.4)$ $(p<0.01)$. In some cases, residual fragments of undigested tissue containing follicles were found with both protocols (Fig. 1d).

\section{Follicle viability}

Shortly after isolation, a total of 237 follicles were analyzed for viability (115 and 122 follicles with the previously applied and modified protocols respectively). The

Table 1 Follicle yield according to follicle isolation protocol. Individual follicle yield from 22 patients according to different follicle isolation protocols

\begin{tabular}{|c|c|c|}
\hline Patient & Established & Modified \\
\hline 1 & 2 & 11 \\
\hline 2 & 23 & 93 \\
\hline 3 & 45 & 61 \\
\hline 4 & 162 & 117 \\
\hline 5 & 152 & 272 \\
\hline 6 & 50 & 105 \\
\hline 7 & 110 & 90 \\
\hline 8 & 0 & 0 \\
\hline 9 & 341 & 483 \\
\hline 10 & 200 & 303 \\
\hline 11 & 7 & 18 \\
\hline 12 & 13 & 19 \\
\hline 13 & 105 & 177 \\
\hline 14 & 125 & 135 \\
\hline 15 & 58 & 70 \\
\hline 16 & 7 & 28 \\
\hline 17 & 29 & 65 \\
\hline 18 & 17 & 15 \\
\hline 19 & 105 & 91 \\
\hline 20 & 65 & 119 \\
\hline 21 & 125 & 127 \\
\hline 22 & 197 & 324 \\
\hline Total & 1938 & 2732 \\
\hline
\end{tabular}

percentage of viable and minimally damaged follicles (V1 and V2) was high and similar between the two isolation protocols $(85 \%$ and $96 \%$ for old and new respectively). Hence, the percentage of damaged and dead follicles (V3 and V4) was low and also comparable (15\% and $4 \%$ respectively). The number of live follicles (V1 and V2) was therefore significantly greater than the number of dead follicles with both isolation protocols $(p<0.0001)$.

\section{After encapsulation}

\section{Follicle counting and classification}

A total of 22 fibrin clots $(n=10$ with the previous protocol and $n=12$ with the modified protocol) were processed for hematoxylin and eosin staining and compared according to follicle developmental stage (primordial, primary or secondary). As shown in Table 2, the modified protocol yielded significantly higher number of primordial and primary follicles $(p<0.05)$ and a significantly lower number of secondary follicles $(p<0.01)$ than the previous protocol. Primordial, primary and secondary follicles are illustrated after isolation and fibrin encapsulation in Fig. 2.

\section{Follicle health status}

Of 25 follicles (previous protocol $n=9$; modified protocol $n=16$ ) analyzed for caspase-3, none were positive, which proves that $100 \%$ of evaluated follicles were alive soon after isolation and encapsulation, irrespective of isolation protocol (Fig. 3a, b).

A total of 44 follicles were analyzed for Ki67 (previous protocol $n=29$; modified protocol $n=15$ ). With the previous protocol, 8 follicles $(27.6 \%)$ were Ki67 positive, $3.4 \%$ of which were primordial $(\mathrm{n}=1), 10.3 \%$ primary $(n=3)$, and $13.8 \%$ secondary follicles $(n=4)$ (Fig. 3c). With the modified protocol, only 1 follicle $(6.7 \%)$ was found to be positive for Ki67 and it was at the primarysecondary stage (Fig. 3d). However, no difference was found in the number of Ki67 positive follicles between the two protocols (Table 3).

\section{After grafting Follicle recovery rate}

Since around $50 \%$ of encapsulated follicles are found soon after fibrin polymerization and histological investigation $[21,25]$, in order to evaluate the real number of follicles remaining after transplantation, we applied a correction factor as previously reported [21, 25]. Consequently, 18 out of 50 follicles (35\%) were identified on day 7 of transplantation. Of this number, $72 \%$ and $28 \%$ were at the primordial and primary stage respectively. Figure 4 shows some of these recovered follicles after 7 days of grafting. 


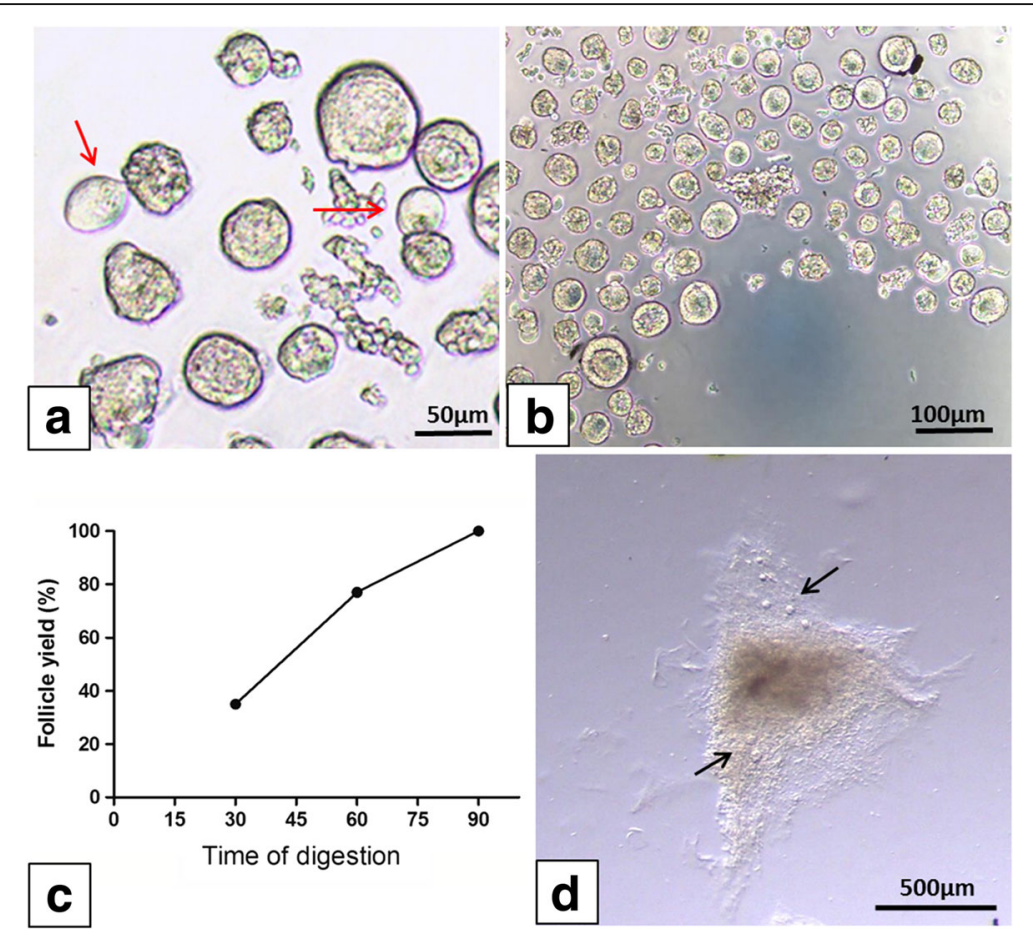

Fig. 1 Isolation of human preantral follicles. In the previous protocol, some follicles showed extruded oocytes (red arrow) (a); in the modified protocol, the vast majority of follicles were isolated from ovarian tissue samples (b). Distribution of isolated follicles in the modified protocol according to the three time intervals (c); some follicles remained entrapped in fragments of undigested tissue (black arrows) (d)

\section{Discussion}

Aiming to create a transplantable artificial ovary, our goal was to optimize our previous follicle isolation protocol in order to increase the number and survival of isolated human preantral follicles $[9,10]$. While the previous method does yield a high number of isolated preantral follicles, we always observed extruded oocytes $[9,10]$ and undigested fragments of tissue. This was probably due to the prolonged and fixed duration of enzymatic digestion (75 min). For this reason, we hypothesized that a successful isolation protocol should take into account two important points: first, the high degree of heterogeneity in follicle density, not only between women and age groups [13, 25], but even between fragments of ovarian tissue from the same patient [14]; and

Table 2 Follicle developmental stage after fibrin matrix encapsulation. Follicle developmental stages were compared between the previous and modified isolation protocols

\begin{tabular}{lcc}
\hline \multicolumn{3}{l}{ Number of follicle stage after fibrin encapsulation } \\
\hline Primordial & $14(7 \%)^{a}$ & Modified protocol (\%) \\
Primary & $127(66 \%)^{a}$ & $90(77 \%)^{b}$ \\
Secondary & $53(27 \%)^{c}$ & $9(8 \%)^{d}$ \\
\hline
\end{tabular}

Different letters $(a, b, c, d)$ in each row indicate significant differences $(a, b$ $p<0.05 ; \mathrm{c}, \mathrm{d} p<0.01)$ second, the variations in extracellular matrix composition between women of different age $[15,16]$ and health status $[15,18]$ and even between different areas of the same ovarian tissue sample $[16,27]$, which influences success rates of the isolation procedure. To meet these requirements, the follicle isolation protocol should be tailored to each patient; instead of a fixed period for enzymatic digestion, as currently reported in the literature [7-11], this step would be halted as soon as all the fragments are totally digested. In addition, enzymatic digestion would be divided into several time intervals, in order to recover already isolated follicles, which would reduce the proportion of damaged follicles due to prolonged digestion. Therefore, with a view to maximizing the yield and quality of isolated ovarian follicles for each patient, we developed a tailored follicle isolation protocol that addresses the two limitations encountered with our earlier method $[9,10]$, namely long and fixed enzyme exposure time. Comparison of the two isolation protocols showed that with our modifications, based on fractioning enzymatic digestion over three different durations, we could obtain a greater number of isolated follicles than with the previously applied protocol. Since $35 \%$ of isolated follicles were already identified after the initial $30 \mathrm{~min}$ of enzymatic digestion in the modified protocol, we can confirm our hypothesis that the long period of enzyme exposure with the previous method 


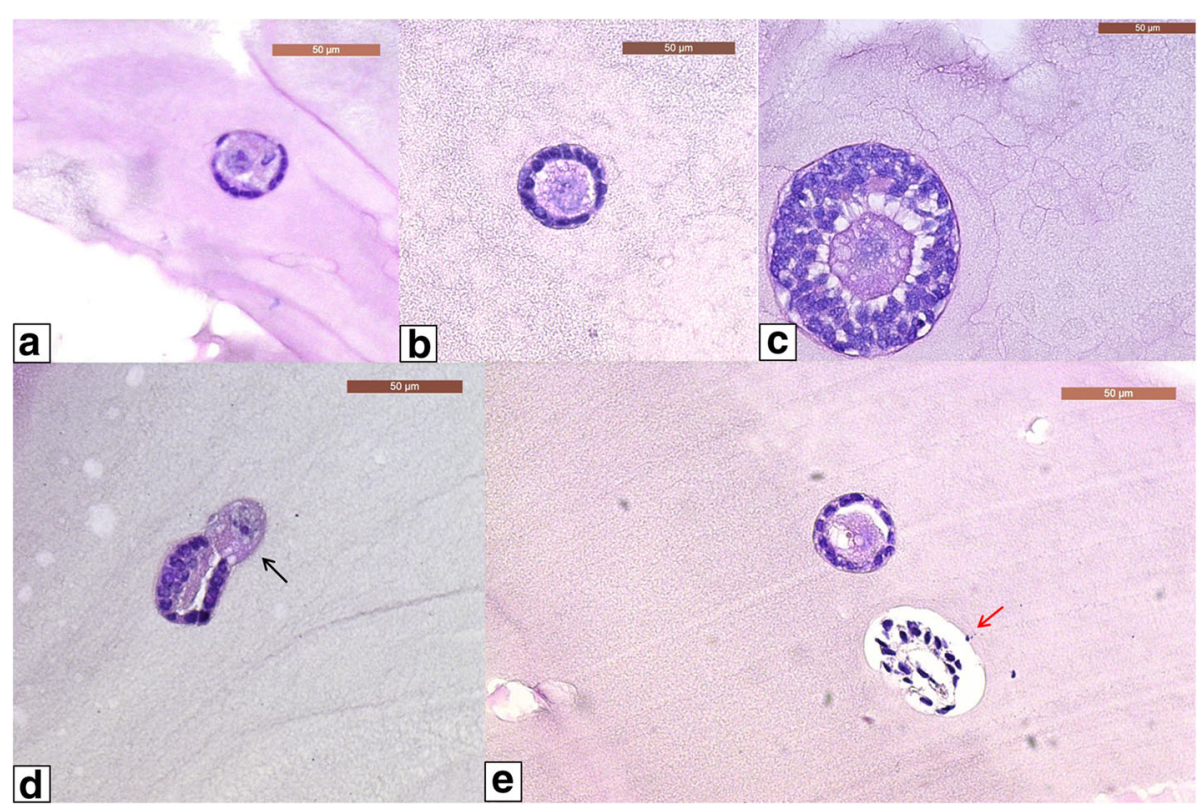

Fig. 2 Histologically normal representations of human follicles encapsulated in a fibrin matrix. Primordial (a), primary (b) and secondary (c) follicles after isolation with the modified protocol and encapsulation in a fibrin matrix. An extruded oocyte (black arrow) (d) and normal and degenerated follicles (red arrow) were found with the previous protocol after histological analysis (e)

has a negative impact on follicles. Although dispase present in high concentrations in Liberase DH does not cleave laminin [28], which is one of the main components of the basement membrane of human primordial follicles [29], it cleaves collagen IV [28], another essential constituent of the basement membrane of these follicles [29]. Hence, one can assume that follicles isolated during the first few minutes of enzymatic digestion could have their basement membrane progressively damaged over the course of $75 \mathrm{~min}$ of exposure to the enzyme, which would probably explain the presence of extruded oocytes in our previous studies $[9,10]$. As also demonstrated by histological analysis, the number of primordial and primary follicles was significantly higher after fibrin encapsulation in the modified protocol (92.3\%) than in the earlier one (73.4\%), which more closely reflects the physiological distribution of follicles inside ovarian cortex $[19,30]$. Our findings therefore confirm that discontinuous enzymatic digestion positively affects recovery rates of isolated follicles by protecting their structure from unnecessary exposure to Liberase $\mathrm{DH}$. Interestingly, the percentage of secondary follicles was greater

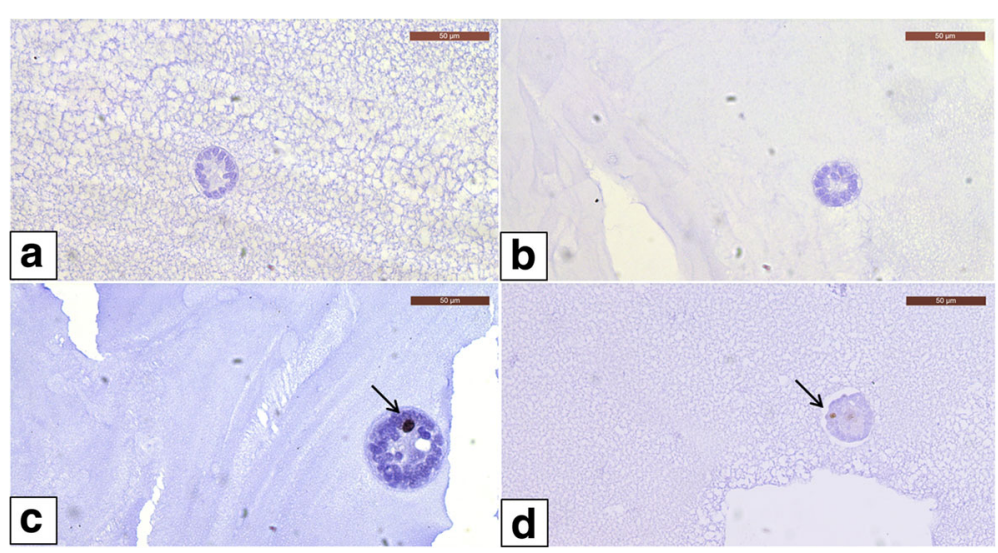

Fig. 3 Follicle health status after caspase-3 and Ki67 immunohistochemistry. Caspase-3-negative primary follicles isolated with the established (a) and modified (b) isolation protocols. Ki67-positive follicles isolated with the established (c) and modified (d) isolation protocols 
Table 3 Distribution of Ki67-positive and -negative follicles according to the established and modified isolation protocols. Follicle developmental stages were compared between the previous and modified isolation protocols

\begin{tabular}{|c|c|c|c|c|c|}
\hline \multirow[t]{2}{*}{ Follicle stage } & \multicolumn{2}{|c|}{ Established $(n=29)$} & \multicolumn{2}{|c|}{ Modified $(n=15)$} & \multirow[t]{2}{*}{$p$-value } \\
\hline & Ki67 + & Ki67 - & $\mathrm{Ki} 67+$ & Ki67 - & \\
\hline \multirow[t]{2}{*}{ Primordial } & $1^{a}$ & 28 & $0^{\mathrm{a}}$ & 15 & 1.000 \\
\hline & $(3.4 \%)$ & & $0 \%$ & & \\
\hline \multirow[t]{2}{*}{ Primary } & $3^{b}$ & 26 & $0^{\mathrm{b}}$ & 15 & 0.5402 \\
\hline & (10.3\%) & & $0 \%$ & & \\
\hline \multirow[t]{2}{*}{ Secondary } & $4^{c}$ & 25 & $1^{c}$ & 14 & 0.6467 \\
\hline & (13.8\%) & & $6.7 \%$ & & \\
\hline
\end{tabular}

Different letters $(a, b, c)$ in each row indicate significant differences $(a, b$, $c p<0.05)$

with the previous method than the modified version (26\% versus 9\%), but similar to an earlier report [12]. Unlike in the previous protocol, a filtration step was also added to the new protocol in order to replace follicle centrifugation and separate follicles from ovarian cell suspensions. We could hypothesize that the different percentage of secondary follicles found between the two protocols may be ascribed to some secondary follicles remaining trapped inside the filter and thus getting lost. With the modified isolation protocol, we even recovered a significant number of viable isolated follicles after
90 min of enzymatic incubation, which shows that the ovarian tissue fragments that remained undigested in our previous protocol are actually an important but as yet unexploited source of primordial follicles. Since every follicle counts towards increasing the likelihood of pregnancy in patients and the possibility of repeating this strategy, discarding such precious material may have a negative impact on artificial ovary outcomes. Another important point to bear in mind is that the artificial ovary will be mostly applied to prepubertal cancer patients. The ovaries of these young girls have a softer cortex than that of adult women, since the ovaries accumulate collagen fibers with age [31, 32] making the cortex stiffer. It would therefore be logical to assume that follicle isolation should be considerably faster in young patients than in adult women [13], so it is likely that the 75 -min digestion time in our previous protocol would damage a significant number of these follicles. In order to confirm the effectiveness of the modified isolation protocol for our artificial ovary prototype, one fibrin clot containing isolated follicles and SCs was grafted for a short period of time. Interestingly, with the modified protocol, the superior follicle recovery rate after grafting (35\%) was better than in a previous study (20-23\%), where follicles were isolated using the earlier protocol and grafted inside a fibrin-based matrix [21] for one week. Moreover, in the present study, a higher percentage of

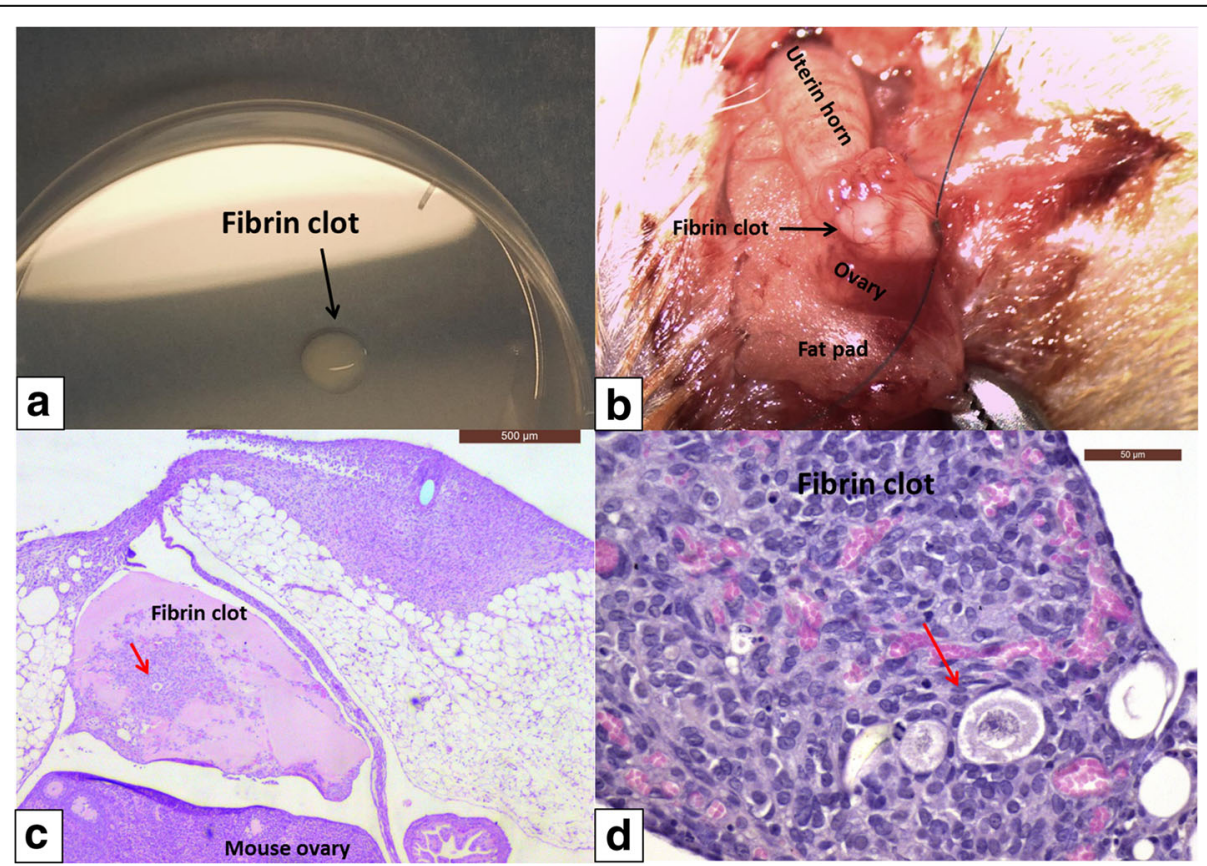

Fig. 4 Grafting of a fibrin clot to the ovarian bursa of a SCID mouse. A fibrin clot containing human follicles and ovarian SCs isolated using the modified protocol before transplantation (a); xenografting of the fibrin clot to the ovarian bursa of a SCID mouse (b); histological representation of the fibrin clot inside the ovarian bursa of a SCID mouse after 7 days of transplantation: a follicle is visible in the middle of the fibrin clot (red arrow) (c); histological representation of follicles found in fibrin clot after 7 days of transplantation (red arrow) (d) 
primordial follicles $(72 \%$ versus $15 \%)$ but a similar proportion of primary follicles ( $28 \%$ versus $26 \%$ ) were found on day 7 of transplantation compared to our previous findings [21]. Given the superior preservation of the primordial follicle pool with the modified protocol, it could be interesting to further investigate whether this protocol plays a role in post-grafting activation of follicles.

\section{Conclusions}

In conclusion, this study describes a personalized isolation procedure for human preantral follicles, which will allow us to maximize the number and quality of isolated primordial and primary follicles. Indeed, fractionating enzymatic digestion yielded a greater number of recovered follicles. Moreover, this protocol can be adapted to each patient, taking into account the density of their ovarian tissue. Therefore, with a view to clinical application of artificial ovary transplantation, we suggest systematic implementation of a tailored follicle isolation approach.

\section{Additional file}

Additional file 1: Supplementary material. (DOCX 18 kb)

\section{Abbreviations}

3D: Three-dimensional; F: Fibrinogen; GC: Granulosa cell; HIFBS: Heatinactivated fetal bovine serum; PBS: Phosphate-buffered saline; RT: Room temperature; SC: Ovarian stromal cell; T: Thrombin

\section{Acknowledgments}

The authors thank Patricia Meijers for her collaboration and scientific advice on fibrin in the project. They also thank Mira Hryniuk, BA, for reviewing the English language of the manuscript, and Dolores Gonzalez and Olivier Van Kerk for their technical assistance.

\section{Funding}

This study was supported by grants from the Fonds National de la Recherche Scientifique de Belgique (FNRS) awarded to C. A. Amorim as a research associate at the FRS-FNRS and M.M. Dolmans (grant 5/4/150/5), FNRS-PDR 0077.14, Fonds Spéciaux de Recherche, Fondation St Luc, Foundation Against Cancer, and Wallonie-Bruxelles International, and donations from the Ferrero family.

\section{Availability of data and materials}

Some of the datasets used and/or analyzed during the current study are available from the corresponding author upon reasonable request.

\section{Authors' contributions \\ MCC and CAA were responsible for the study design and manuscript preparation. MCC, MH, AC and CAA performed the experimental procedures and acquired the data. MMD and JD assisted in revising the manuscript and added critical feedback. All the authors are aware of this submission and approved the final manuscript.}

\section{Ethics approval and consent to participate}

Use of human ovarian tissue was approved by the Institutional Review Board of the Université Catholique de Louvain on 2 June, 2014 (IRB reference 2012/ 23MAR/125, registration number B403201213872).

\section{Consent for publication}

All the authors approved this submission and gave their consent for publication.

\section{Competing interests}

The authors declare that they have no competing interests.

\section{Publisher's Note}

Springer Nature remains neutral with regard to jurisdictional claims in published maps and institutional affiliations.

\section{Author details}

${ }^{1}$ Pôle de Recherche en Gynécologie, Institut de Recherche Expérimentale et Clinique, Université Catholique de Louvain, Avenue Mounier 52, bte. B1.52.02, 1200 Brussels, Belgium. ${ }^{2}$ Gynecology Department, Cliniques Universitaires Saint-Luc, 1200 Brussels, Belgium. ${ }^{3}$ Society for Research into Infertility, Brussels, Belgium

Received: 23 July 2017 Accepted: 10 October 2017

Published online: 23 October 2017

\section{References}

1. Donnez J, Dolmans MM. Fertility preservation in women. Nat Rev Endocrinol. 2013;9(12):735-49.

2. Dolmans MM, Luyckx V, Donnez J, Andersen CY, Greve T. Risk of transferring malignant cells with transplanted frozen-thawed ovarian tissue. Fertil Steril. 2013:99(6):1514-22.

3. Amorim CA, Shikanov A. The artificial ovary: current status and future perspectives. Future oncology (London, England). 2016:12(20):2323-32.

4. Donnez J, Dolmans MM. Ovarian cortex transplantation: 60 reported live births brings the success and worldwide expansion of the technique towards routine clinical practice. J Assist Reprod Genet. 2015;32(8):1167-70.

5. Meirow D, Ra'anani H, Shapira M, Brenghausen M, Derech Chaim S, Aviel-Ronen S, et al. Transplantations of frozen-thawed ovarian tissue demonstrate high reproductive performance and the need to revise restrictive criteria. Fertil Steril. 2016;106(2):467-74.

6. Jensen AK, Macklon KT, Fedder J, Ernst E, Humaidan P, Andersen CY. 86 successful births and 9 ongoing pregnancies worldwide in women transplanted with frozen-thawed ovarian tissue: focus on birth and perinatal outcome in 40 of these children. J Assist Reprod Genet. 2017:34(3):325-36.

7. Amorim CA. Artificial Ovary. In: Gonadal tissue cryopreservation in fertility preservation. Suzuki N, Donnez J, editors. Tokyo: Springer. doi.10.1007/978-4431-55963-4

8. Oktay K, Nugent D, Newton H, Salha O, Chatterjee P, Gosden RG. Isolation and characterization of primordial follicles from fresh and cryopreserved human ovarian tissue. Fertil Steril. 1997;67(3):481-6.

9. Dolmans MM, Michaux N, Camboni A, Martinez-Madrid B, Van Langendonckt A, Nottola SA, et al. Evaluation of Liberase, a purified enzyme blend, for the isolation of human primordial and primary ovarian follicles. Human reproduction (Oxford, England). 2006;21(2):413-20.

10. Vanacker J, Camboni A, Dath C, Van Langendonckt A, Dolmans MM, Donnez J, et al. Enzymatic isolation of human primordial and primary ovarian follicles with Liberase $\mathrm{DH}$ : protocol for application in a clinical setting. Fertil Steril. 2011;96(2):379-83. e3

11. Dong FL, Ma L, Shi SL, Dai SJ, Liu XG, YC S, et al. An research on the isolation methods of frozen-thawed human ovarian preantral follicles. Int J Clin Exp Med. 2014;7(8):2298-303.

12. Lierman S, Tilleman $\mathrm{K}$, Cornelissen M, De Vos WH, Weyers S, T'Sjoen G, et al. Follicles of various maturation stages react differently to enzymatic isolation: a comparison of different isolation protocols. Reprod BioMed Online. 2015;30(2):181-90

13. Soares M, Sahrari K, Amorim CA, Saussoy P, Donnez J, Dolmans MM. Evaluation of a human ovarian follicle isolation technique to obtain diseasefree follicle suspensions before safely grafting to cancer patients. Fertil Steril. 2015;104(3):672-80. e2

14. Soares M, Saussoy P, Maskens M, Reul H, Amorim CA, Donnez J, et al. Eliminating malignant cells from cryopreserved ovarian tissue is possible in leukaemia patients. Br J Haematol. 2017;178:231-39.

15. Delgado-Rosas F, Gaytan M, Morales C, Gomez R, Gaytan F. Superficial ovarian cortex vascularization is inversely related to the follicle reserve in normal cycling ovaries and is increased in polycystic ovary syndrome. Human reproduction (Oxford, England). 2009;24(5):1142-51.

16. Schmidt KL, Byskov AG, Nyboe Andersen A, Muller J, Yding Andersen C. Density and distribution of primordial follicles in single pieces of cortex 
from 21 patients and in individual pieces of cortex from three entire human ovaries. Human reproduction (Oxford, England). 2003;18(6):1158-64.

17. Rodgers RJ, Irving-Rodgers HF, Russell DL. Extracellular matrix of the developing ovarian follicle. Reproduction (Cambridge, England). 2003;126(4):415-24.

18. Woodruff TK, Shea LDA. New hypothesis regarding ovarian follicle development: ovarian rigidity as a regulator of selection and health. J Assist Reprod Genet. 2011;28(1):3-6.

19. Amorim CA, Van Langendonckt A, David A, Dolmans MM, Donnez J. Survival of human pre-antral follicles after cryopreservation of ovarian tissue, follicular isolation and in vitro culture in a calcium alginate matrix. Human reproduction (Oxford, England). 2009;24(1):92-9.

20. Martinez-Madrid B, Dolmans MM, Langendonckt AV, Defrere S, Van Eyck AS, Donnez J. Ficoll density gradient method for recovery of isolated human ovarian primordial follicles. Fertil Steril. 2004:82(6):1648-53.

21. Paulini F, Vilela JM, Chiti MC, Donnez J, Jadoul P, Dolmans MM, et al. Survival and growth of human preantral follicles after cryopreservation of ovarian tissue, follicle isolation and short-term xenografting. Reprod BioMed Online. 2016;33(3):425-32.

22. Cohen GM. Caspases: the executioners of apoptosis. The Biochemical journal. 1997;326(Pt 1):1-16.

23. Scholzen T, Gerdes J. The Ki-67 protein: from the known and the unknown. J Cell Physiol. 2000;182(3):311-22.

24. Chiti MC, Dolmans MM, Orellana R, Soares M, Paulini F, Donnez J, et al, Influence of follicle stage on artificial ovary outcome using fibrin as a matrix. Human reproduction (Oxford, England). 2016;31(2):427-35.

25. Chiti MC, Dolmans MM, Lucci CM, Paulini F, Donnez J, Amorim CA. Further insights into the impact of mouse follicle stage on graft outcome in an artificial ovary environment. Mol Hum Reprod. 2017;

26. Gougeon A. Regulation of ovarian follicular development in primates: facts and hypotheses. Endocr Rev. 1996;17(2):121-55.

27. Kristensen SG, Rasmussen A, Byskov AG, Andersen CY. Isolation of pre-antral follicles from human ovarian medulla tissue. Human reproduction (Oxford, England). 2011;26(1):157-66.

28. Stenn KS, Link R, Moellmann G, Madri J, Kuklinska E. Dispase, a neutral protease from bacillus polymyxa, is a powerful fibronectinase and type IV collagenase. The Journal of investigative dermatology. 1989:93(2):287-90.

29. Heeren AM, van Iperen L, Klootwijk DB, de Melo Bernardo A, Roost MS, Gomes Fernandes MM, et al. Development of the follicular basement membrane during human gametogenesis and early folliculogenesis. BMC Dev Biol. 2015;15:4

30. Gougeon A. Human ovarian follicular development: from activation of resting follicles to preovulatory maturation. Ann Endocrinol. 2010;71(3):132-43.

31. Herraiz S, Diaz-Garcia C, Pellicer A. Ovarian tissue cryopreservation: slow freezing. In: Suzuki N, Donnez J, editors. Gonadal tissue cryopreservation in fertility preservation. Tokyo: Springer Japan; 2016. p. 53-77.

32. Smitz J, Dolmans MM, Donnez J, Fortune JE, Hovatta O, Jewgenow K, et al. Current achievements and future research directions in ovarian tissue culture, in vitro follicle development and transplantation: implications for fertility preservation. Hum Reprod Update. 2010;16(4):395-414.

\section{Submit your next manuscript to BioMed Central and we will help you at every step:}

- We accept pre-submission inquiries

- Our selector tool helps you to find the most relevant journal

- We provide round the clock customer support

- Convenient online submission

- Thorough peer review

- Inclusion in PubMed and all major indexing services

- Maximum visibility for your research

Submit your manuscript at www.biomedcentral.com/submit

CBiomed Central 